04

\title{
Микроволновая диагностика разрядов в искусственном облаке заряженных водяных капель
}

\author{
() Н.А. Богатов, ${ }^{2}$ В.С. Сысоев, ${ }^{1}$ Д.И. Сухаревский, ${ }^{1}$ М.Ю. Наумова ${ }^{1}$ \\ ${ }^{1}$ Российский фредеральный ядерный центр - Всероссийский научно-исследовательский институт \\ технической фризики им. акад. Е.И. Забабахина, \\ 143500 Истра, Московская обл., Россия \\ ${ }^{2}$ Федеральный исследовательский центр Институт прикладной физики РАН, \\ 603950 Нижний Новгород, Россия \\ e-mail: v.s.sysoev@vniitf.ru
}

Поступило в Редакцию 1 ноября 2021 г.

В окончательной редакции 22 ноября 2021 г.

Принято к публикации 26 ноября 2021 г.

\begin{abstract}
Разработана и создана микроволновая диагностика разрядов, возникающих в искусственном облаке заряженных водяных капель, моделирующем среду грозовых облаков. Ослабление зондирующего микроволнового излучения с длиной волны $8 \mathrm{~mm}$, проходящего через искусственное электризованное облако, измерялось с временным разрешением $10 \mathrm{~ns}$. С помощью созданной микроволновой диагностики были исследованы временные характеристики внутриоблачных разрядов.
\end{abstract}

Ключевые слова: искра, лидер, стример, электромагнитная волна.

DOI: $10.21883 /$ JTF.2022.03.52133.284-21

\section{Введение}

До сих пор молния остается недостаточно изученным природным явлением, несмотря на двухсотлетнюю историю ее интенсивных исследований. Традиционные методы исследования молнии, а также длинных искровых разрядов, во многих аспектах моделирующих молнию, основаны на регистрации видимого излучения, испускаемого этими разрядами [1-3]. Однако в облачной среде видимое излучение сильно рассеивается, поэтому разрядные процессы внутри грозовых облаков оказываются недоступными традиционным методам наблюдения молниевых разрядов. Натурные экспериментальные исследования непосредственно внутри грозовых облаков сопряжены с большими техническими трудностями, и поэтому очень редки и имеют ограниченные возможности. Для изучения разрядных процессов в облачной среде в лабораторных условиях создаются искусственные облака заряженных водяных капель $[4,5]$, электризованных настолько, что между этими облаками и заземленными поверхностями, а также внутри облаков, возникают электрические разряды. Два таких генератора заряженных облаков в настоящее время работают во ВНИЦ РФЯЦ-ВНИИТФ. Средний радиус капель в этих облаках около $0.5 \mu \mathrm{m}$. Такие капли эффективно рассеивают свет видимого диапазона, поэтому применение традиционных в физике искровых разрядов оптических методов исследования для изучения разрядов внутри таких облаков невозможно по той же причине, по которой остаются неизученными разряды внутри грозовых облаков, - разряды не видны. В работах [6-8] для наблюдения разрядов внутри искусственного облака использовалась инфракрасная (ИК) камера со спектральным диапазоном чувствительности 2.5-5.5 больше размера капель искусственного облака, поэтому рассеяние ИК излучения не столь значительно, как для видимого света. Поэтому ИК изображения внутриоблачных разрядов были успешно получены. Недостатком этого метода исследования внутриоблачных разрядов была невысокая скорость ИК камеры. Время экспозиции (2-3 ms) значительно превышало длительность всего разрядного процесса, поэтому невозможно было увидеть динамику разряда. В настоящей работе для исследования внутриоблачных разрядов была использована микроволновая диагностика с высоким временным разрешением. Микроволновое излучение практически не рассеивается каплями микронного размера, но может поглощаться и рассеиваться плазменными образованиями, возникающими при разрядных процессах внутри облака. Интенсивность собственного микроволнового излучения электроразрядной плазмы недостаточна для надежной регистрации в условиях высокого уровня электромагнитных помех, характерного для высоковольтного эксперимента. Это заставляет использовать активную микроволновую диагностику, просвечивая облако микроволновым пучком. Необходимо отметить, что исследования длинных искровых разрядов с применением активных методов диагностики немногочисленны. Для регистрации пониженной плотности газа в искровых каналах вследствие нагрева газа применялся шлиренметод [9-17] и интерферометрический метод [18-20]. В работе [21] активный метод микроволновой диагностики был впервые применен для исследования лабораторного длинного искрового разряда, возбуждаемого в 
промежутке стержень-плоскость мегавольтным импульсом напряжения. В настоящей работе микроволновая диагностика, аналогичная примененной в [21], была реализована и использована для исследования длинных искровых разрядов внутри лабораторного искусственного электризованного облака. Созданная в данной работе система микроволнового зондирования искусственного облака имела временное разрешение $10 \mathrm{~ns}$. Использование этой диагностики позволило получить временные характеристики развития внутриоблачных разрядов.

\section{1. Описание эксперимента и СВЧ диагностики}

Схема эксперимента приведена на рис. 1. Искусственное облако формировалось в результате конденсации водяного пара, поступающего из парогенератора через сопло, расположенное в центре заземленного плоского электрода диаметром $3 \mathrm{~m}$. Электризация образующихся в потоке пара капель производилась посредством коронного разряда на игольчатом электроде, расположенном в сопле. Подробное описание установки для создания искусственного заряженного облака дано в [6]. Средний радиус капель, оцененный на основании измерений в работе [22], в наших экспериментах составлял около $0.5 \mu \mathrm{m}$ [6]. Заряд облака, при котором начинаются разряды, по оценке составлял около $60 \mu \mathrm{C}$ [6]. Другие параметры облака, такие, как концентрация капель и распределение капель по зарядам и массам, неизвестны.

В настоящей работе была использована самая простая для реализации схема микроволновой диагностики регистрировалось ослабление микроволнового пучка, проходящего через облако с ионизацией. Источником микроволнового излучения служил генератор Г4-91, нагруженный на рупорную антенну. Генератор работал в непрерывном режиме. Выходная мощность генератора

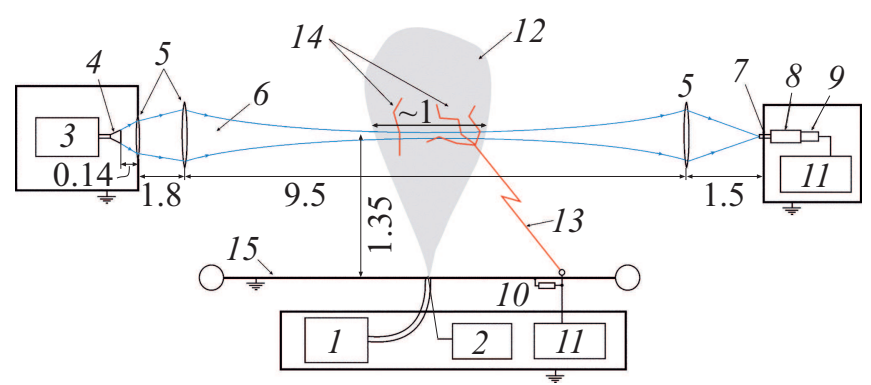

Рис. 1. Схема эксперимента и микроволновой диагностики (расстояния указаны в метрах): 1 - парогенератор, 2 - высоковольтный источник питания, 3 - СВЧ генератор, 4 - рупорная антенна, 5- линзы, 6 - СВЧ пучок, 7 - приемный волновод, 8 - СВЧ усилитель $20 \mathrm{~dB}, 9-$ СВЧ детектор, 10 - измерительный омический малоиндуктивный шунт, 11 - осциллограф, 12 - заряженное аэрозольное облако, 13 - искровой разряд облако-земля, $14-$ разряды (и части разрядов) внутри облака, 15 - заземленная плоскость. составляла $5 \mathrm{~mW}$, частота излучения - $37 \mathrm{GHz}$, уровень флуктуаций выходной мощности $-10^{-3}$. Сходящийся гауссовый микроволновой пучок формировался диэлектрическими линзами так, чтобы перетяжка пучка находилась приблизительно в середине облака в месте наиболее интенсивных разрядов. Диаметр микроволнового пучка в перетяжке на $1 / e$ уровне интенсивности составлял $\sim 10 \mathrm{~cm}$ (распределение интенсивности в поперечном сечении пучка в перетяжке, близкое к гауссовскому, было измерено СВЧ диодом). Форма микроволнового пучка между визуально видимыми границами облака была почти цилиндрической (на границах облака расчетная ширина пучка была на $3 \%$ больше, чем в перетяжке). Расстояние от оси микроволнового пучка до заземленной плоскости равнялось $1.35 \mathrm{~m}$. Поляризация микроволнового излучения была линейной, перпендикулярной заземленной плоскости. Прошедший через облако микроволновой пучок фокусировался линзой на открытый конец приемного волновода, затем усиливался $20 \mathrm{~dB}-$ усилителем и детектировался СВЧ диодом Д404 с линейной вольт-ваттной характеристикой и постоянной времени $10 \mathrm{~ns}$. Выходной сигнал с СВЧ диода регистрировался осциллографом. Особенностью высоковольтного эксперимента является наличие большой электромагнитной наводки на измерительное оборудование во время искрового разряда, поэтому необходимо было тщательно экранировать как излучательную, так и приемную части измерительной аппаратуры. Для этого СВЧ генератор помещался в экранированном помещении, а приемная часть аппаратуры была помещена в закрытый металлический контейнер с автономным электропитанием для исключения прохождения помехи по проводам.

Ослабление микроволнового излучения, вызываемое облаком в отсутствие электрической активности, было ниже порога чувствительности нашей диагностики. Относительное ослабление микроволнового излучения в облаке определялось как разность единицы и отношения выходного сигнала СВЧ диода к его уровню в отсутствие облака или в отсутствие электрической активности в облаке.

Чувствительность нашей микроволновой диагностики определялась уровнем флуктуаций выходной мощности СВЧ генератора. Минимальный уровень регистрируемого относительного ослабления микроволнового излучения, прошедшего через облако, составлял $10^{-3}$.

\section{2. Ослабление СВЧ излучения в области, занятой искровым разрядом}

Длинные искровые разряды всегда образуют протяженные плазменные каналы приблизительно круглого сечения, диаметр которых много меныше их длины. Поэтому рассеяние и поглощение некоторым участком 
канала искрового разряда электромагнитной волны, падающей на искровой канал, можно полагать приблизительно таким же, как у круглого цилиндра бесконечной длины и такого же диаметра, как плазменный канал. Каналы длинных искровых разрядов ориентированы преимущественно вдоль электрического поля. В нашем эксперименте электрическое поле заряженного облака и разряды в нем были ориентированы преимущественно по нормали к заземленной плоскости, приблизительно так же, как и поляризация зондирующего микроволнового излучения. Поэтому взаимодействие микроволнового излучения с плазменными каналами внутриоблачных разрядов в нашем эксперименте ближе всего к задаче дифракции плоской линейно поляризованной электромагнитной волны на круглом бесконечном плазменном цилиндре, ось которого параллельна вектору электрического поля в волне.

\section{1. Поглощение СВЧ излучения стримерами}

Задача дифракции плоской линейно поляризованной электромагнитной волны на круглом цилиндре слабоионизованной воздушной плазмы (вектор электрического поля волны параллелен оси цилиндра) была рассмотрена в [21]. Было показано, что если в атмосферном воздухе при нормальных условиях радиус плазменного цилиндра $r$ и концентрация электронов в нем $n$ удовлетворяют условию

$$
n r^{2} \ll 1.2 \cdot 10^{13} \mathrm{~cm}^{-1},
$$

то справедливы следующие утверждения:

1) интенсивность поглощения электромагнитной волны миллиметрового диапазона много больше интенсивности рассеяния;

2) амплитуда рассеянной волны на поверхности цилиндра много меньше амплитуды падающей волны, поэтому амплитуда высокочастотного электрического поля на поверхности цилиндра приблизительно равна амплитуде падающей волны;

3) толщина скин-слоя внутри цилиндра много больше его радиуса, поэтому электрическое поле внутри цилиндра приблизительно однородное и равно полю падающей волны.

Характерные параметры плазмы стримерных каналов [1], $n \leq 10^{14} \mathrm{~cm}^{-3}, r \leq 1 \mathrm{~mm}$, удовлетворяют условию (1), поэтому ослабление микроволнового излучения, проходящего через стримерный разряд, приблизительно равно мощности, поглощаемой всеми свободными электронами стримеров, находящимися в падающем микроволновом пучке. Мощность, поглощаемая одним свободным электроном в газе, находящимся в высокочастотном электрическом поле с амплитудой $E$ равна [23]

$$
w_{e}=\frac{e^{2} v_{e} E^{2}}{2 m\left(v_{e}^{2}+\omega^{2}\right)},
$$

где $\omega-$ круговая частота поля, $v_{e}-$ частота транспортных столкновений электронов с молекулами воздуха, $e$ и $m$ - заряд и масса электрона соответственно. При условии $v_{e} \gg \omega$, которое при частоте $37 \mathrm{GHz}$ в воздухе нормальной плотности выполняется, выражение (2) принимает вид

$$
w_{e}=\frac{e^{2} E^{2}}{2 m v_{e}} .
$$

В искровых разрядах в плотных газах и, в частности, в атмосфере стримеры, как правило, развиваются одновременно в большом количестве. Например, в стримерной зоне лидера объемом порядка кубического метра одновременно присутствуют $10^{5}-10^{6}$ стримеров [1]. Мощность $W_{a}$ поглощения микроволнового излучения на единичной площади поперечного сечения пучка, проходящего через область, занятую стримерами с характерным размером, много большим радиуса микроволнового пучка, при условии сохранения цилиндричности микроволнового пучка (постоянстве амплитуды поля) равна

$$
W_{a}=\frac{e^{2} E^{2}}{2 m v_{e}} n_{e s}, \quad n_{e s}=\int n_{e} d z
$$

где $n_{e s}$ - интеграл концентрации электронов вдоль оси микроволнового пучка. Относительное ослабление микроволнового излучения, проходящего через область, занятую стримерами, равно отношению удельной (на единицу площади пучка) мощности, поглощаемой в стримерах, к плотности потока мощности в падающем микроволновом пучке $S_{0}=0.5 \sqrt{\varepsilon_{0} / \mu_{0}} E^{2}$ :

$$
\delta=\frac{W_{a}}{S_{0}}=\frac{e^{2} n_{e s}}{m v_{e}} \sqrt{\frac{\mu_{0}}{\varepsilon_{0}}} .
$$

Уравнение (5) дает связь величины $n_{e s}$ с измеряемой величиной ослабления зондирующего микроволнового излучения $\delta$ :

$$
n_{e s}=2.8 \cdot 10^{13} \delta \mathrm{cm}^{-2} \text {. }
$$

Стримерным разрядам, состоящим, как правило, из множества одновременно живущих стримеров, появляющимся на разных этапах развития искровых разрядов или развивающимся самостоятельно, и являющимся частью общего разрядного процесса, часто свойственно состоять из приблизительно одинаковых стримеров. Например, таким свойством обладает стримерная зона лидеров в фазе свободного движения лидера и начале сквозной фазы [21]. В этом случае величину $n_{e s}$ можно представить следующим образом:

$$
n_{e s}=N_{e} \int n_{s t r} d z
$$

где $N_{e}$ - полное число свободных электронов в одном стримере, а $n_{s t r}-$ концентрация стримеров в пространстве. Если из независимых измерений можно найти концентрацию стримеров $n_{s t r}$ и ее распределение в пространстве, то по измерению относительного ослабления микроволнового излучения $\delta$ можно, используя (6) и (7), найти полное количество электронов в одном стримере $N_{e}$ (как это было сделано в [21]). 
Оценим величину $\delta$ в случае, когда все облако (размер облака вдоль микроволнового пучка $L \sim 1 \mathrm{~m}$ ) заполнено стримерами, имеющими такие же параметры и такую же концентрацию, как стримеры в стримерной зоне положительного лидера, [21]: $N_{e}=3 \cdot 10^{10}, n_{s t r}=0.1 \mathrm{~cm}^{-3}$. Тогда получим

$$
n_{e s} \sim 3 \cdot 10^{11} \mathrm{~cm}^{-2}, \quad \delta \sim 0.01 .
$$

\section{2. Рассеяние СВЧ излучения длинными искровыми каналами}

Проводимость лидерных каналов на четыре порядка величины выше проводимости стримерных каналов [1]. Взаимодействие электромагнитной волны с такими каналами подобно дифракции на идеально проводящем цилиндре. В этом случае поглощение микроволнового излучения в плазме таких каналов мало, а ослабление проходящего излучения обусловлено рассеянием на каналах. Из решения задачи о дифракции плоской монохроматической электромагнитной волны на бесконечном круглом идеально проводящем цилиндре [24] следует, что амплитуда электрического поля $E_{r}$ в отраженной цилиндрической волне равна

$$
\begin{aligned}
E_{r}= & E_{0}\left[\frac{J_{0}(k a)}{H_{0}^{(2)}(k a)} H_{0}^{(2)}(k r)\right. \\
& \left.+2 \sum_{m=1}^{\infty} \frac{J_{m}(k a)}{H_{m}^{(2)}(k a)} H_{m}^{(2)}(k r) \cos (m \varphi)\right],
\end{aligned}
$$

где $E_{0}$ - амплитуда падающей плоской волны, $a-$ радиус цилиндра, $k-$ волновое число падающей волны, $J_{m}-$ функция Бесселя, $H_{m}^{(2)}-$ функция Ганкеля второго рода, $r$ и $\varphi-$ цилиндрические координаты. Эффективный радиус рассеяния $d_{\mathrm{eff}}$, равный отношению рассеянной мощности на единицу длины цилиндра $W_{r}$ к плотности потока в падающей волне $S_{i}$, равен

$$
\begin{aligned}
d_{\mathrm{eff}}=\frac{W_{r}}{S_{i}} & =\left.\frac{1}{\left|E_{i}\right|^{2}} \int_{0}^{2 \pi}\left|E_{r}\right|^{2} r d \varphi\right|_{r \rightarrow \infty} \\
& =\frac{4}{k}\left\{\left|\frac{J_{0}(k a)}{H_{0}^{(2)}(k a)}\right|^{2}+2 \sum_{m=1}^{\infty}\left|\frac{J_{m}(k a)}{H_{m}^{(2)}(k a)}\right|^{2}\right\} .
\end{aligned}
$$

На рис. 2 приведена зависимость отношения эффективного диаметра рассеяния к диаметру цилиндра от отношения диаметра цилиндра к длине электромагнитной волны, полученная из формулы (10).

Из (10) легко найти часть $\delta$ мощности монохроматического гауссова пучка электромагнитных волн с радиусом $R$ и волновым числом $k$, рассеиваемую на проводящем цилиндре радиусом $a \ll R$, расположенном перпендикулярно оси пучка на расстоянии $b$ от нее:

$$
\delta=\frac{4}{\sqrt{\pi} k R} e^{-\frac{b^{2}}{R^{2}}}\left\{\left|\frac{J_{0}(k a)}{H_{0}^{(2)}(k a)}\right|^{2}+2 \sum_{m=1}^{\infty}\left|\frac{J_{m}(k a)}{H_{m}^{(2)}(k a)}\right|^{2}\right\} .
$$

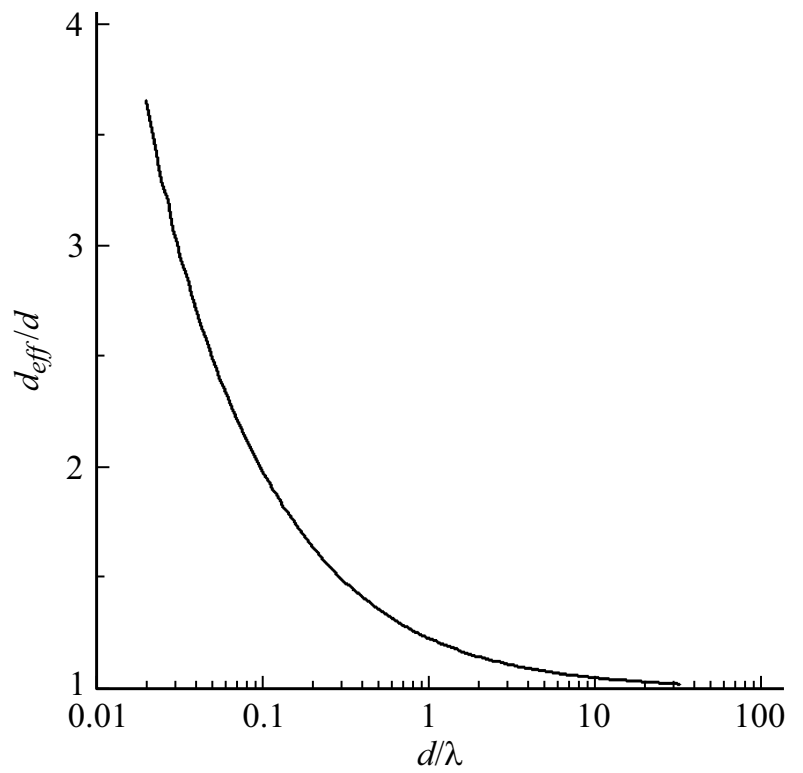

Рис. 2. Отношение эффективного диаметра рассеяния к диаметру идеально проводящего цилиндра в зависимости от отношения диаметра цилиндра к длине волны.

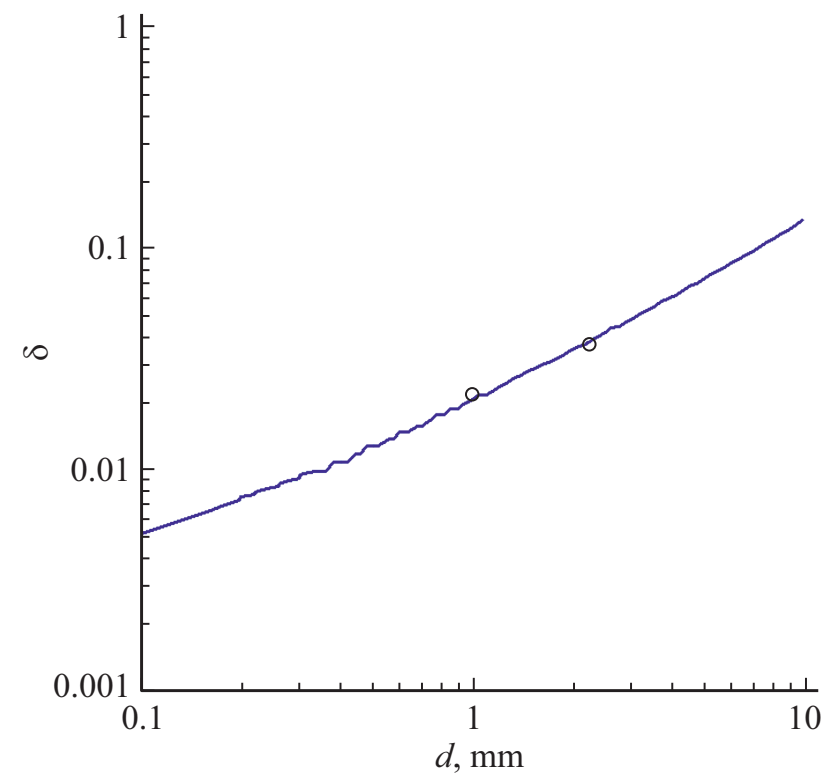

Рис. 3. Относительное ослабление микроволнового излучения с частотой $37 \mathrm{GHz}$ в пучке радиусом $R=5 \mathrm{~cm}$ при дифракции на проводящем цилиндре диаметром $d$, расположенном на оси микроволнового пучка и ориентированном вдоль направления поляризации пучка, в зависимости от диаметра цилиндра. Кружки - эксперимент с металлическими цилиндрами.

На рис. 3 показана зависимость относительного ослабления $\delta$ от диаметра цилиндра для частоты электромагнитных волн в пучке $37 \mathrm{GHz}$ при расположении цилиндра на оси пучка $(b=0)$, рассчитанная по формуле (11). Кружками на рис. 3 показаны результаты тестовых измерений относительного ослабления при помещении 
в перетяжке микроволнового пучка металлических проводов диаметрами 1 и $2.25 \mathrm{~mm}$.

\section{3. Некоторые результаты измерения ослабления микроволнового излучения в искусственном облаке во время внутриоблачных разрядов}

В относительном ослаблении СВЧ излучения во время электрических разрядов в облаке наблюдались два характерных вида сигналов. Один из них - это интенсивные короткие пики, амплитуда которых составляла обычно несколько процентов (самые интенсивные до $10 \%$ ), а длительность $0.3-0.4 \mu \mathrm{s}$. Другая форма относительного поглощения - это плавные горбы, амплитуда которых была около $1 \%$ и меньше, а длительность несколько десятков микросекунд. В начале плавных горбов всегда наблюдался интенсивный пик, в то время как пики могли появляться в одиночестве. На рис. 4 приведена запись относительного ослабления микроволнового излучения, на которой присутствуют обе формы [25]. Исследования внутриоблачных разрядов [26], проведенные с использованием микроволновой диагностики совместно со съемкой ИК камерой и электрическими измерениями, показали, что резкие пики ослабления зондирующего СВЧ-излучения вызваны стримерными вспышками, развивающимися в облаке. Плавные горбы, вероятно, связаны со стримерными зонами лидеров, которые в наших экспериментах обычно зарождались на заземленной плоскости и двигались к облаку. Величина поглощения иногда превышала оценку (8), сделанную выше для параметров стримерной зоны положительного лидерного разряда. Это значит, что стримерные разряды в электризованном облаке могут быть более интенсивными, т. е. иметь более высокую концентрацию стримеров или концентрацию электронов в стримерах,

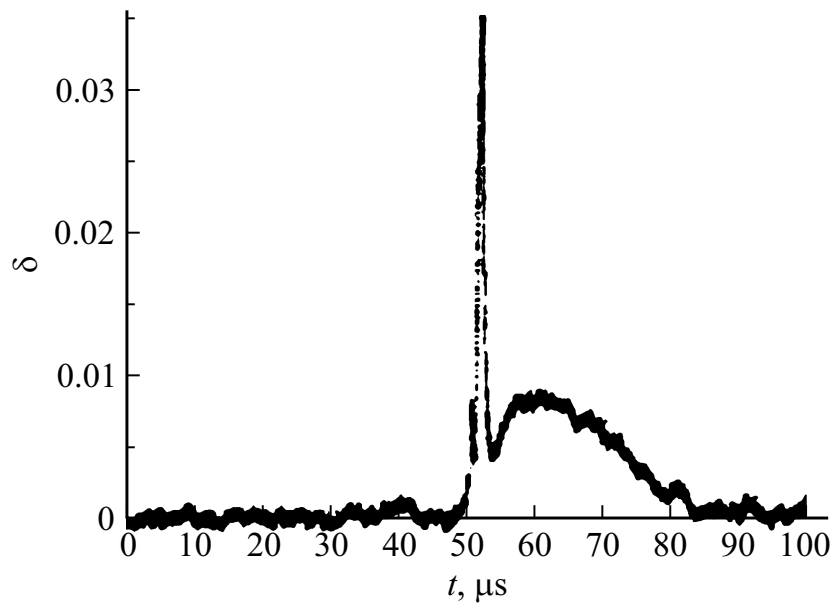

Рис. 4. Относительное ослабление $\delta$ микроволнового излучения, проходящего через электризованное облако, во время протекания внутри облака электрических разрядов (адаптировано из [25]). чем стримерные зоны лидеров положительных длинных искровых разрядов.

\section{Заключение}

СВЧ диагностика является эффективным инструментом исследования электрических разрядов в облачной среде, которая позволяет получать информацию о параметрах внутриоблачных разрядов, недоступную традиционным оптическим методам, обычно используемым в искровых разрядных экспериментах.

Полученные результаты могут быть полезны при исследованиях распространения СВЧ излучения через атмосферные зоны с грозовой активностью для оценки интенсивности поглощения и рассеяния СВЧ излучения разрядами внутри грозовых облаков.

\section{Благодарность}

Исследование выполнено за счет гранта Российского научного фонда (проект № 19-19-00501). Авторы благодарны двум рецензентам за полезные замечания и комментарии, позволившие заметно улучшить качество статьи.

\section{Конфликт интересов}

Авторы заявляют, что у них нет конфликта интересов.

\section{Список литературы}

[1] Э.М. Базелян, Ю.П. Райзер. Искровой разряд (МФТИ, M., 1997)

[2] Э.М. Базелян, Ю.П. Райзер. Физика молнии и молниезащиты (Физматлит, М., 2001)

[3] V.A. Rakov, M.A. Uman. Lightning: Physics and Effects (Cambridge Univ. Press, NY., 2003)

[4] K.V. Antsupov, I.P. Vereshchagin, M.A. Koshelev, L.M. Makalsky, V.S. Syssoev. Discharges from Cloud of Charged Aerosol, in Proc. 7th Int. Symp. on High Voltage Engineering (Tech. Univ. of Dresden, Dresden, Germany, 1991), p. 15-17.

[5] А.Г. Темников, Л.Л. Черненский, А.В. Орлов, Т.К. Герастенок. Вестник МЭИ, 4, 75 (2013).

[6] A.Yu. Kostinskiy, V.S. Syssoev, N.A. Bogatov, E.A. Mareev, M.G. Andreev, L.M. Makalsky, D.I. Sukharevsky, V.A. Rakov. Geophys. Res. Lett., 42, 8165 (2015). DOI: 10.1002/2015GL065620 (Supporting Information).

[7] A.Y. Kostinskiy, V.S. Syssoev, N.A. Bogatov, E.A. Mareev, M.G. Andreev, M.U. Bulatov, L.M. Makal'sky, D.I. Sukharevsky, V.A. Rakov. J. Geophys. Res. Atmos., 121 (16), 9756 (2016). DOI: 10.1002/2016JD025079

[8] V.A. Rakov, E.A. Mareev, M.D. Tran, Y. Zhu, N.A. Bogatov, A.Yu. Kostinskiy, V.S. Syssoev. IEEJ Transactions on Electrical and Electronic Engineering, 138 (5), 321 (2018). DOI: $10.1541 /$ ieejpes.138.321

[9] Les Renardieres Group. Electra, 53, 31 (1977).

[10] A. Kurimoto, O. Farish, D.J. Tedford. Proceedings Institution of Electrical Engineers, 125 (8), 767 (1978). 
[11] Les Renardières Group. Electra, 1981 (74), 67 (1981).

[12] A. Gibert, J. Dupuy, M. Bayle, P. Bayle. J. Phys. D: Appl. Phys., 16 (8), 1493 (1983). DOI: 10.1088/0022-3727/16/8/016

[13] P. Domens, J. Dupuy, A. Gibert, R. Diaz, B. Hutzler, J.P. Riu, F. Ruhling. J. Phys. D: Appl. Phys., 21, 1613 (1988). DOI: $10.1088 / 0022-3727 / 21 / 11 / 011$

[14] P. Domens, A. Gibert, J. Dupuy, F. Ruhling. J. Phys. D: Appl. Phys., 24 (7), 1088 (1991). DOI: 10.1088/0022-3727/24/7/010

[15] P. Ortega, P. Domens, A. Gibert, B. Hutzler, G. Riquel. J. Phys. D: Appl. Phys., 27, 2379 (1994). DOI: $10.1088 / 0022-3727 / 27 / 11 / 019$.

[16] T. Reess, P. Ortega, A. Gibert, P. Domens, P. Pignolet. J. Phys. D: Appl. Phys., 28 (11), 2306 (1995).

DOI: $10.1088 / 0022-3727 / 28 / 11 / 011$

[17] S. Larigaldie. J. Appl. Phys., 61, 90 (1987). DOI: $10.1063 / 1.338806$

[18] T. Fukuchi, K. Nemoto. High-Speed Shadowgraphy and Interferometry Using an Acousto-Optic Laser Deflector, Proc. SPIE 5920, Ultrafast X-Ray Detectors, HighSpeed Imaging and Applications, 59200R (21 September 2005). DOI: $10.1117 / 12.616256$

[19] X. Zhou, R. Zeng, C. Zhuang, S. Chen. Phys. Plasmas, 22, 063508 (2015). DOI: $10.1063 / 1.4922660$

[20] Y. Cui, R. Zeng, C. Zhuang, X. Zhou, Z. Wang, S. Chen. arXiv:1801.04664[physics.app-ph]

[21] N.A. Bogatov, A.Y. Kostinskiy, V.S. Syssoev, M.G. Andreev, M.U. Bulatov, D.I. Sukharevsky, E.A. Mareev, V.A. Rakov. J. Geophysical Research: Atmospheres, 123, e2019JD031826 (2020). DOI: 10.1029/2019JD031826

[22] Т.С. Басиев, И.П. Верещагин, Л.М. Макальский, Г.З. Мирзабекян, В.И. Савченко, В.С. Сысоев, В.В. Ушаков, Г.М. Франчук. Генераторы заряженного аэрозоля Известия АН СССР Энергетика и транспорт, 5, 127 (1982).

[23] Ю.П. Райзер. Физика газового разряда (Наука, Физматлит, М., 1992)

[24] S. Solimeno, B. Crosignani, P. Di Porto. Guiding, Diffraction and Confinement of Optical Radiation (Academic Press, Orlando, 1986)

[25] N.A. Bogatov, V.S. Syssoev, D.I. Suharevsky, M.U. Bulatov, M.G. Andreev, A.Yu. Kostinsky, E.A. Mareev, V.A. Rakov. Microwave Diagnostics for Investigation of Long Spark and Artificial Charged Aerosol Cloud, Proc. 15-th Int. Conf. On Atmospheric Electricity, June 15-20, 2014, Norman, Oklahoma, USA.

[26] A.Yu. Kostinskiy, N.A. Bogatov, V.S. Syssoev, E.A. Mareev, M.G. Andreev, M.U. Bulatov, D.I. Sukharevsky, V.A. Rakov. Unusual Plasma Formations Produced by Positive Streamers Entering the Cloud of Negatively Charged Water Droplets, preprint Earth and Space Science Open Archive. DOI: 10.1002/essoar.10508088.1 